\title{
Reference Values for External and Internal Training Intensity Monitoring in Young Male Soccer Players: A Systematic Review
}

\author{
Rafael Oliveira ${ }^{1,2,3, * \mathbb{C}}$, João Paulo Brito ${ }^{1,2,3} \mathbb{\infty}$, Adrián Moreno-Villanueva ${ }^{4} \mathbb{C}^{\text {, Matilde Nalha }}{ }^{1}(\mathbb{D}$, \\ Markel Rico-González ${ }^{5}\left(\mathbb{D}\right.$ and Filipe Manuel Clemente ${ }^{6,7}$ (D)
}

1 Sports Science School of Rio Maior, Polytechnic Institute of Santarém, 2140-413 Rio Maior, Portugal; jbrito@esdrm.ipsantarem.pt (J.P.B.); matildenalha@gmail.com (M.N.)

2 Research Center in Sport Sciences, Health Sciences and Human Development, 5001-801 Vila Real, Portugal

3 Life Quality Research Centre, 2140-413 Rio Maior, Portugal

4 Department of Physical Activity and Sport Sciences, International Excellence Campus "Mare Nostrum", Faculty of Sports Sciences, University of Murcia, 30720 San Javier, Spain; more_adri@hotmail.com

5 Department of Didactics of Musical, Plastic and Corporal Expression, University of the Basque Country, UPV-EHU, 48940 Leioa, Spain; markeluniv@gmail.com

6 Escola Superior Desporto e Lazer, Instituto Politécnico de Viana do Castelo, Rua Escola Industrial e Comercial de Nun'Álvares, 4900-347 Viana do Castelo, Portugal; filipe.clemente5@gmail.com

7 Instituto de Telecomunicações, Delegação da Covilhã, 1049-001 Lisboa, Portugal

* Correspondence: rafaeloliveira@esdrm.ipsantarem.pt

Citation: Oliveira, R.; Brito, J.P.; Moreno-Villanueva, A.; Nalha, M.; Rico-González, M.; Clemente, F.M. Reference Values for External and Internal Training Intensity Monitoring in Young Male Soccer Players: A Systematic Review. Healthcare 2021, 9, 1567. https:// doi.org/10.3390/healthcare9111567

Academic Editor: Parisi Attilio

Received: 20 October 2021

Accepted: 11 November 2021

Published: 17 November 2021

Publisher's Note: MDPI stays neutral with regard to jurisdictional claims in published maps and institutional affiliations.

Copyright: (c) 2021 by the authors. Licensee MDPI, Basel, Switzerland. This article is an open access article distributed under the terms and conditions of the Creative Commons Attribution (CC BY) license (https:// creativecommons.org/licenses/by/ $4.0 /)$.

\begin{abstract}
Training intensity monitoring is a daily practice in soccer which allows soccer academies to assess the efficacy of its developmental interventions and management strategies. The current systematic review's purpose is to: (1) identify and summarize studies that have examined external and internal training intensity monitoring, and to (2) provide references values for the main measures for young male soccer players. A systematic review of EBSCO, PubMed, Scielo, Scopus, SPORTDiscus, and Web of Science databases was performed according to the Preferred Reporting Items for Systematic Reviews and Meta-Analyses (PRISMA) guidelines. From the 2404 studies initially identified, 8 were fully reviewed, and their outcome measures were extracted and analyzed. From them, the following range intervals were found for training: rated perceived exertion (RPE) 2.3-6.3 au; session$\mathrm{RPE}, 156-394 \mathrm{au}$; total distance, 3964.5-6500 $\mathrm{m}$ and; distance $>18 \mathrm{~km} / \mathrm{h}, 11.8-250 \mathrm{~m}$. Additionally, a general tendency to decrease the intensity in the day before the match was Found. This study allowed to provide reference values of professional young male players for the main internal and external measures. All together, they can be used by coaches, their staff, or practitioners in order to better adjust training intensity.
\end{abstract}

Keywords: football; male; training; youth; RPE; GPS; running; high-speed running; sprint

\section{Introduction}

Training intensity monitoring is a daily practice within the soccer training context [1]. Training intensity describes how hard a player is exercising [2]. Understanding the impact of training stimulus on the players and identify the variations between players contributes to an adjustment of planning and for adjusting specific recovery or managing strategies [3]. For that reason, coaches expect that training intensity monitoring guides them for planning better for an improvement in performance and reduction in injury risk [1]. Quantifying aspects of young players development and performance (e.g., physical abilities, physiological capacities, technical and tactical skill) allows soccer academies to assess the efficacy of its developmental interventions and management strategies [4]. By collecting and analyzing regular inputs related to player development and performance, organizations create a feedback loop in which their planning and training interventions can be objectively assessed.

The monitoring is commonly organized in two dimensions [5]: (i) internal intensity; and (ii) external intensity. The internal intensity represents the psychophysiological impact 
of external intensity on the player's organism while the external intensity represents the mechanical and physical impact of training drills on the players [6]. Different instruments can be used to assess these dimensions, however in soccer, the most common instruments for measuring internal intensity are heart rate monitors and rated perceived exertion (RPE) scale, while for measuring the external intensity the most common are microelectromechanical systems (e.g., global positioning system, local positioning system, inertial measurement units) [7].

From such instruments, the typical variables extracted are heart rate-bases scores, RPEbased scores and distances covered at different speed thresholds, or changes of velocity (accelerations and decelerations). The measures are daily quantified in training and match scenarios. Using absolute intensity per session and per week, the sports scientist has some possibilities of understanding the within- and between-weeks variations [7].

The analysis of intensity impact on players is highly relevant. One of the reasons is justified by the gap between coaches perception and the actual intensity imposed on the players [8]. Based on that, employing intensity monitoring in youth teams became a typical strategy used by coaches [9]. Thus, a growing number of researches have been published in training monitoring in youth soccer players [10-12].

Although the increase in intensity monitoring reports in young soccer, most of the articles are from the same team. This reduces the possibility of generalization of the evidence. However, is also known that is not expectable to reach different teams at the same time over long periods of the season. Thus, one of the strategies can be to summarize the available evidence about intensity values of young soccer players by conducting a systematic review. Although a possible idea, no systematic review was found about that. Although there is pertinence of looking into both males and females, summarizing both within the same article would not be useful since the huge differences related with a number of publications and a logical difference between sexes produces a different approach to the consequences for the article logic. Thus, a more focused contribution as a systematic review may help coaches to compare values of training intensity in young male soccer and provide some benchmarks. Thus, the aim of this systematic review is to identify and summarize studies that have examined external and internal training monitoring and to provide references values for the main measures for young male soccer players.

\section{Materials and Methods}

The preferred reporting items for systematic reviews and meta-analyses (PRISMA) guidelines were followed to write this systematic review [13] and guidelines for performing systematic reviews in sport sciences [14]. The protocol of the systematic review was a priori registered in the International Platform of Registered Systematic Review and Meta-Analysis Protocols with the number INPLASY202180055 and the DOI number 10.37766/inplasy2021.8.0055.

\subsection{Eligibility Criteria}

The inclusion and exclusion criteria can be found in Table 1.

The screening process related to analysis of the title, abstract and reference list of each study to locate potentially relevant studies was independently executed by two of the authors (A.M.V. and M.R.G.). Moreover, both authors also reviewed the full version of the included papers in detail to identify which article met the inclusion criteria. Additionally, a search within the reference lists of the included records was performed to add additional relevant studies. In the cases of discrepancies, a discussion was performed with the participation of a third author (R.O.). Possible errata for the included articles were considered. 
Table 1. Eligibility criteria.

\begin{tabular}{|c|c|c|}
\hline PICOS & Inclusion Criteria & Exclusion Criteria \\
\hline Population & $\begin{array}{l}\text { Healthy young (under eighteen) male } \\
\text { soccer players. }\end{array}$ & $\begin{array}{l}\text { Studies conducted with professional or amateur } \\
\text { players or in other sports, or with } \\
\text { female populations. }\end{array}$ \\
\hline Intervention/Exposure & $\begin{array}{l}\text { Exposure to entire training sessions for number } \\
\text { of weeks and sessions included (minimum } \\
\text { one week). }\end{array}$ & $\begin{array}{l}\text { No exposure to entire training sessions (e.g., } \\
\text { specific exercises only reported; only matches; only } \\
\text { simulated matches). }\end{array}$ \\
\hline Comparator & $\begin{array}{l}\text { Not required. Eventually, comparisons between } \\
\text { playing positions and/or competitive levels } \\
\text { within the same age-group and/or age-groups. }\end{array}$ & No study will be excluded based on comparators. \\
\hline Outcomes & $\begin{array}{l}\text { Presents at least of one measure among the } \\
\text { included in internal (e.g., heart rate, rate of } \\
\text { perceived exertion) and/or external intensity } \\
\text { (e.g., distances covered at different speed } \\
\text { thresholds, acceleration-based measures) in } \\
\text { absolute values. Whenever relative values } \\
\text { allow to calculate absolute values, the study } \\
\text { will be included. }\end{array}$ & $\begin{array}{l}\text { Absence of data characterizing the intensity } \\
\text { during the training sessions (e.g., wellness } \\
\text { variables, readiness parameters) and or only } \\
\text { reports the data in relative values without } \\
\text { allowing the calculation of absolute values. Data } \\
\text { from workload calculations will also be excluded } \\
\text { (e.g., accumulated weekly intensity, training } \\
\text { monotony, strain, ACWR, EWMA). }\end{array}$ \\
\hline Study design & No restrictions imposed on study design. & $\begin{array}{l}\text { No study was excluded on the basis of } \\
\text { study design. }\end{array}$ \\
\hline Others & $\begin{array}{l}\text { Only original and full-text studies written } \\
\text { in English. }\end{array}$ & $\begin{array}{l}\text { Written in other language than English. Other } \\
\text { article types than original (e.g., reviews, letters to } \\
\text { editors, trial registrations, proposals for protocols, } \\
\text { editorials, book chapters and conference abstracts). }\end{array}$ \\
\hline
\end{tabular}

PICOS: (P) population; (I) intervention/exposure; (C) comparator; (O) outcomes; (S) study design.

\subsection{Information Sources}

The following electronic databases were used to search for relevant publication on the 3 July 2021, after protocol registration: FECYT (MEDLINE, Scielo, and Web of Science), PubMed, and Scopus. A manual search was also conducted after search in electronic databases to retrieve additional studies that could fit our eligibility criteria.

\subsection{Search Strategy}

Keywords and synonyms were entered in various combinations in the title, abstract or keywords which means that the following research content was applied: ("soccer" OR "football") AND ("internal load" OR "external load" OR "workload" OR "training load" OR "load monitoring"). Search results were exported to EndNote 20.0.1 for Mac (Clarivate Analytics). No filters or limits were applied.

\subsection{Data Extraction}

A specific spreadsheet was designed in Microsoft Excel (Microsoft Corporation, Readmon, WA, USA) for process the data extraction. The design followed the recommendations of the Cochrane Consumers and Communication Review Group's data extraction template [15]. In this spreadsheet the information about inclusion and exclusion requirements and reasons was detailed. The selection of the articles was made independently by two authors (A.M.-V. and M.R.-G.). In the cases of discrepancies, a discussion was performed with the participation of a third author (R.O.).

\subsection{Methodological Assessment}

The methodological quality was assessed using the methodological index for nonrandomized studies (MINORS) by two authors (A.M.-V. and M.R.-G.) [16]. MINORS consists of twelve items, four of which are only applicable to comparative studies which was not the case of the included studies. Thus, only eight items were applied. Each item is rated 
as 0 when the criterion is not reported in the article, 1 if reported but not sufficiently fulfilled, or 2 when adequately met. Higher scores indicate good methodological quality of the article and a low risk of bias. The highest possible score is 16 for non-comparative studies. MINORS has yielded acceptable inter- and intra-rater reliability, internal consistency, content validity, and discriminative validity $[16,17]$.

\section{Results}

A total of 2404 (i.e., FECYT: 1481; PubMed: 806; Scopus: 117) original articles were initially retrieved from the mentioned databases, of which 834 were duplicates. Thus, a total of 1570 original articles were found. After this, a total of 1558 articles checked by title and abstract were excluded. The remaining 12 articles were checked in full text, leading to the exclusion of 3 articles according to criterion $\mathrm{n}^{\circ} 1$, and 1 according to criterion $\mathrm{n}^{\circ} 2$. Additionally, 1 article was included from additional sources. A total of 8 articles met all the inclusion criteria and were finally included in the qualitative synthesis. All the steps followed for the selection of the articles are available in Figure 1.

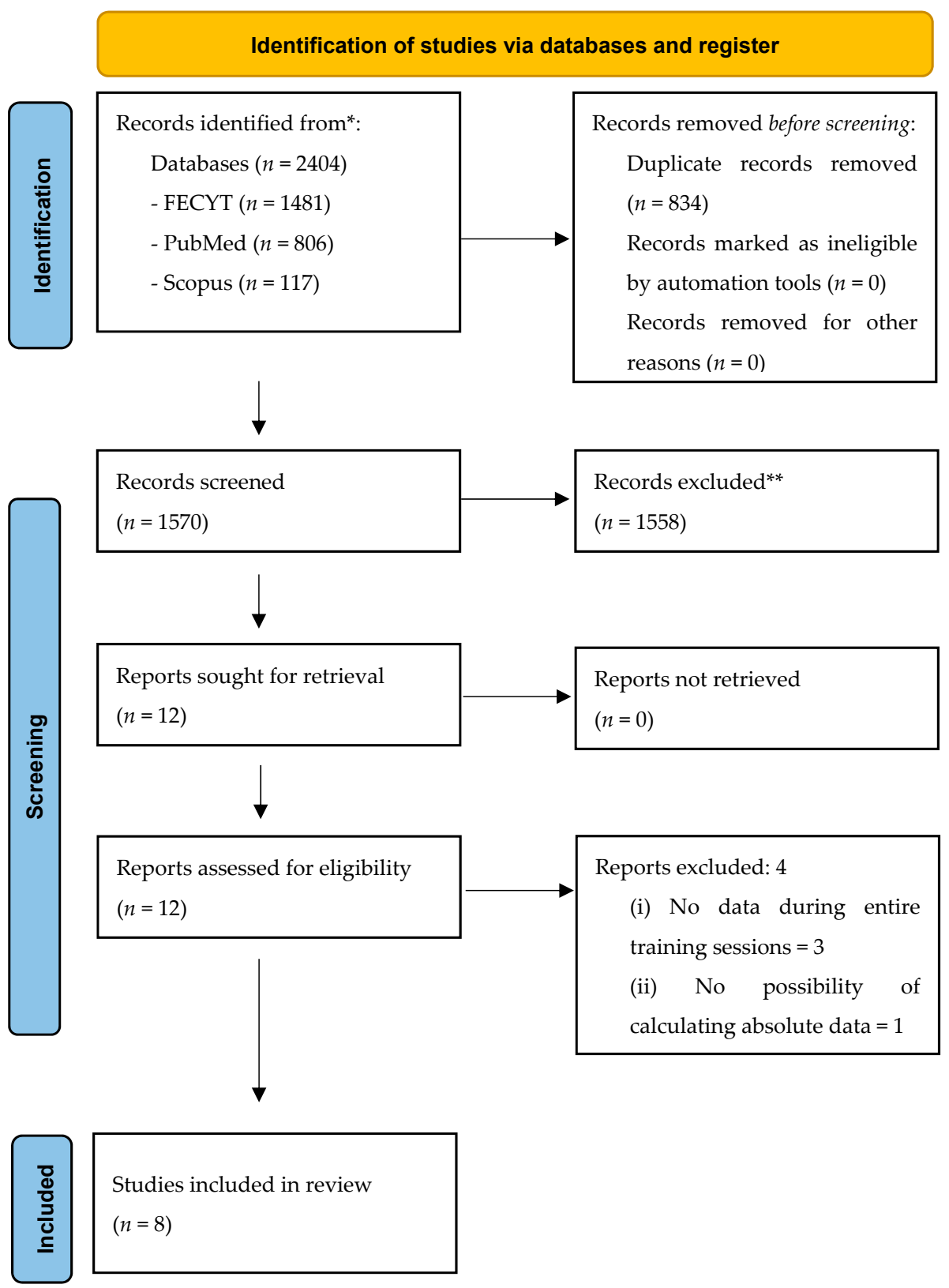

Figure 1. Preferred reporting item for systematic reviews (PRISMA) flow diagram. 


\subsection{Methodological Quality}

The overall methodological quality of the cross-sectional studies can be found in Table 2.

Table 2. Methodological assessment using MINORS checklist.

\begin{tabular}{clllllllll}
\hline Study & $\mathbf{1}$ & $\mathbf{2}$ & $\mathbf{3}$ & $\mathbf{4}$ & $\mathbf{5}$ & $\mathbf{6}$ & $\mathbf{7}$ & $\mathbf{8}$ & Score \\
\hline$[18]$ & 2 & 2 & 1 & 1 & 1 & 2 & 2 & 1 & $12 / 16$ \\
{$[19]$} & 2 & 2 & 2 & 2 & 0 & 1 & 2 & 1 & $12 / 16$ \\
{$[20]$} & 2 & 2 & 1 & 1 & 1 & 2 & 2 & 2 & $13 / 16$ \\
{$[21]$} & 2 & 2 & 2 & 2 & 1 & 2 & 2 & 2 & $15 / 16$ \\
{$[22]$} & 2 & 2 & 2 & 2 & 1 & 1 & 2 & 2 & $14 / 16$ \\
{$[23]$} & 2 & 2 & 1 & 2 & 0 & 2 & 2 & 1 & $12 / 16$ \\
{$[24]$} & 2 & 2 & 2 & 2 & 1 & 2 & 2 & 2 & $15 / 16$ \\
{$[25]$} & 2 & 2 & 2 & 1 & 0 & 1 & 2 & 1 & $11 / 16$ \\
\hline
\end{tabular}

Note: The MINORS checklist asks the following information $(2=$ High quality; 1 = Medium quality; $0=$ Low quality): 1 . Clearly defined objective. 2 . Inclusion of patients consecutively. 3. Information collected retrospectively. 4. Assessments adjusted to objective. 5. Evaluations carried out in a neutral way. 6. Follow-up phase consistent with the objective. 7. Dropout rate during follow-up less than 5\%. 8. Appropriate statistical analysis.

\subsection{Results of the Studies}

Table 3 presents the characteristics of the studies regarding their sample size, their age of the sample, their duration, as well as training duration, internal and external measures, and instruments used. From the eight studies included, the study of Hannon et al. was the only to analyze the categories of under12 (U12), U13 [21]. Two studies analyzed U14 category $[21,25]$. Four studies analyzed U15 category $[18,20,21,24]$. Two studies analyzed the category of U16 [21,25]. Three studies analyzed the category of U17 [18,23,24]. Two studies analyzed the category of U18 [21,25]. Finally, three studies analyzed the category of U19 [18,22,24].

Table 3. Study characteristics.

\begin{tabular}{|c|c|c|c|c|c|}
\hline Study & Sample & Age & $\begin{array}{c}\text { Study and } \\
\text { Training Duration }\end{array}$ & $\begin{array}{l}\text { Internal Measures/ } \\
\text { Instruments }\end{array}$ & $\begin{array}{l}\text { External Measures/ } \\
\text { Instruments }\end{array}$ \\
\hline [18] & $\begin{array}{l}\text { U15 N:56 } \\
\text { U17 N:66 } \\
\text { U19 N:29 }\end{array}$ & $\begin{array}{l}14.0 \pm 0.2 \\
15.8 \pm 0.4 \\
17.8 \pm 0.6\end{array}$ & $\begin{array}{l}9 \text { weeks } \\
\text { Training duration: } 90 \text { min }\end{array}$ & $\begin{array}{c}1 \text { Hz Polar Team } \\
\text { System, Polar, FI: } \\
\text { HR }<75 \% ; \\
\text { HR } 75-84.9 \% \\
\text { HR } 85 \% \text { to } 89.9 \% \\
\text { HR } \geq 90 \%\end{array}$ & $\begin{array}{c}15 \mathrm{~Hz} \text { GPS (SPI-Pro X II, GPSports, } \\
\text { Canberra, Australia): } \\
\text { Total distance } \\
\text { Distance } 0-6.9 \mathrm{~km} / \mathrm{h} \\
\text { Distance } 7.0-9.9 \mathrm{~km} / \mathrm{h} \\
\text { Distance } 10.0-12.9 \mathrm{~km} / \mathrm{h} \\
\text { Distance } 13-15.9 \mathrm{~km} / \mathrm{h} \\
\text { Distance } 16-17.9 \mathrm{~km} / \mathrm{h} \\
\text { Distance } \geq 18.0 \mathrm{~km} / \mathrm{h} \\
\text { Distance } \geq 18.0 \mathrm{~km} / \mathrm{h} \text { NR } \\
\text { Impacts NR }\end{array}$ \\
\hline [19] & U17 N:18 & $15.7 \pm 0.5$ & $\begin{array}{l}38 \text { training sessions } \\
\text { Training duration ND }\end{array}$ & $\begin{array}{l}1 \text { HZ Polar Team Pro } \\
\text { tracking system: HR }\end{array}$ & $\begin{array}{c}10 \mathrm{~Hz} \text { Polar Team Pro tracking } \\
\text { System: } \\
\text { Total distance, } \\
\text { Distance } 11-14.9 \mathrm{~km} / \mathrm{h} \\
\text { Distance } 15.0-18.9 \mathrm{~km} / \mathrm{h} \\
\text { Distance } \geq 19.00 \mathrm{~km} / \mathrm{h} \\
\text { Acceleration } \geq 2.0 \mathrm{~m} \cdot \mathrm{s}^{-2} \mathrm{NR}\end{array}$ \\
\hline [20] & U15 N:22 & $14.5 \pm 0.3$ & $\begin{array}{l}4 \text { weeks } \\
\text { Training duration ND }\end{array}$ & s-RPE (CR-10) & - \\
\hline [21] & $\begin{array}{l}\text { U12 N:15 } \\
\text { U13 N:13 } \\
\text { U14 N:12 } \\
\text { U15 N:10 } \\
\text { U16 N:11 } \\
\text { U18 N:15 }\end{array}$ & $\begin{array}{l}11.7 \pm 0.2 \\
12.6 \pm 0.3 \\
13.7 \pm 0.2 \\
14.5 \pm 0.3 \\
15.5 \pm 0.2 \\
17.0 \pm 0.4\end{array}$ & $\begin{array}{c}1 \text { in-season } \\
\text { Training duration: described in } \\
\text { Table } 5\end{array}$ & - & $\begin{array}{c}10 \mathrm{~Hz} \text { GPS (Apex, STATSports, } \\
\text { Northern Ireland): } \\
\text { Total distance } \\
\text { Distance } 19.8-25.2 \mathrm{~km} / \mathrm{h} \\
\text { Distance }>25 \mathrm{~km} / \mathrm{h}\end{array}$ \\
\hline
\end{tabular}


Table 3. Cont.

\begin{tabular}{|c|c|c|c|c|c|}
\hline Study & Sample & Age & $\begin{array}{c}\text { Study and } \\
\text { Training Duration }\end{array}$ & $\begin{array}{l}\text { Internal Measures/ } \\
\text { Instruments }\end{array}$ & $\begin{array}{l}\text { External Measures/ } \\
\text { Instruments }\end{array}$ \\
\hline [22] & U19 N:9 & $17.6 \pm 0.6$ & $\begin{array}{l}1 \text { in-season } \\
\text { Training duration } 79 \mathrm{~min}\end{array}$ & - & $\begin{array}{l}10 \mathrm{~Hz} \text { GPS OptimEye X4 (Catapult } \\
\text { Sports, Melbourne, Australia): } \\
\text { Total distance } \\
\text { Distance } 12-15 \mathrm{~km} / \mathrm{h} \\
\text { Distance } 15-20 \mathrm{~km} / \mathrm{h} \\
\text { Distance } 20-25 \mathrm{~km} / \mathrm{h} \\
\text { Distance }>25 \mathrm{~km} / \mathrm{h}\end{array}$ \\
\hline [23] & U17 N: 21 & $16.1 \pm 0.2$ & $\begin{array}{c}36 \text { weeks } \\
\text { Training duration ND }\end{array}$ & s-RPE (CR-10) & - \\
\hline [24] & $\begin{array}{l}\text { U15 N:20 } \\
\text { U17 N:20 } \\
\text { U19 N:20 }\end{array}$ & $\begin{aligned} 13.2 & \pm 0.5 \\
15.4 & \pm 0.5 \\
17.39 & \pm 0.55\end{aligned}$ & $\begin{array}{l}2 \text { Weeks } \\
\text { Training duration } 90 \mathrm{~min}\end{array}$ & $\begin{array}{l}\text { RPE (CR-20) } \\
\text { s-RPE (CR-20) }\end{array}$ & $\begin{array}{c}18 \mathrm{~Hz} \text { GPS STATSports Apex }{ }^{\circledR} \text { (Newry, } \\
\text { Northern Ireland): } \\
\text { Total distance } \\
\text { Maximal speed } \\
\text { HMLD }\left(>25.5 \mathrm{~W} \cdot \mathrm{kg}^{-1}\right) \\
\text { Distance }>25 \mathrm{~km} / \mathrm{h} \\
\text { Distance }>25 \mathrm{~km} / \mathrm{h} \mathrm{NR} \\
\text { Accelerations } \geq 3 \mathrm{~m} \cdot \mathrm{s}^{-2} \\
\text { Decelerations } \leq-3 \mathrm{~m} \cdot \mathrm{s}^{-2}\end{array}$ \\
\hline [25] & $\begin{array}{l}\text { U14 N:8 } \\
\text { U16 N:8 } \\
\text { U18 N:8 }\end{array}$ & $\begin{array}{l}13 \pm 1 \\
15 \pm 1 \\
17 \pm 1\end{array}$ & $\begin{array}{c}2 \text { Weeks } \\
\text { Field training duration: } \\
\text { U14-90 min } \\
\text { U16-102 min } \\
\text { U18-104 min } \\
\text { Gym training duration: } \\
\text { 30-35 min }\end{array}$ & $\begin{array}{c}5 \mathrm{~Hz} \text { Polar Team } \\
\text { System }{ }^{\circledR}, \text { Kempele, } \\
\text { Finland): HR } \\
\text { s-RPE (CR10) }\end{array}$ & - \\
\hline
\end{tabular}

U: under; min: minutes; HR: heart rate; NR: number; ND: non described; HMLD: high metabolic load distance; RPE: rated perceived exertion; s-RPE: session rated of perceived exertion.

In addition, three studies analyzed both internal and external measures [18,19,24], three studies only analyzed internal measures $[20,23,25]$ and two studies only analyzed external measures [21,22].

Table 4 presents the results for external measures in which four studies were included $[18,19,22,24]$. The table was organized according to the age categories.

Only one study presented data for starters and non-starters. Since non-starter presented lower values than starters, we provided a range interval with lower values related to non-starters and higher values related to starters [19].

The last line of Table 4 presents the range intervals for the external measures most used.

Table 5 presents the results for external intensity by match-day minus (MD-) and by players positions (last two lines). The approach of MD- was used by two studies [21,24], while player positions was analyzed by one study [24]. The last line of Table 5, before player positions data, presents the range intervals according to the MD- approach for the measures most used.

Table 6 presents results for internal intensity in which one study included 1-match week and 2 matches-week analysis [20], one study analyzed player positions [23], one study analyzed player status (starters vs. non-starters) [19], three studies analyzed the average overall team [24-26] and two studies used MD- approach [23,24]. The sessionrated of perceived exertion (s-RPE) was the variable most used to quantify internal training intensity. Finally, one study presented internal intensity for gym training [25]. The main results showed a range interval range of 2.3 to 6.3 arbitrary units (au) for RPE between U14, U15, U16, and U18 [20,25] and 156-394 au for s-RPE between U15 and U17 [20,23]. 
Table 4. Results for external training by overall team.

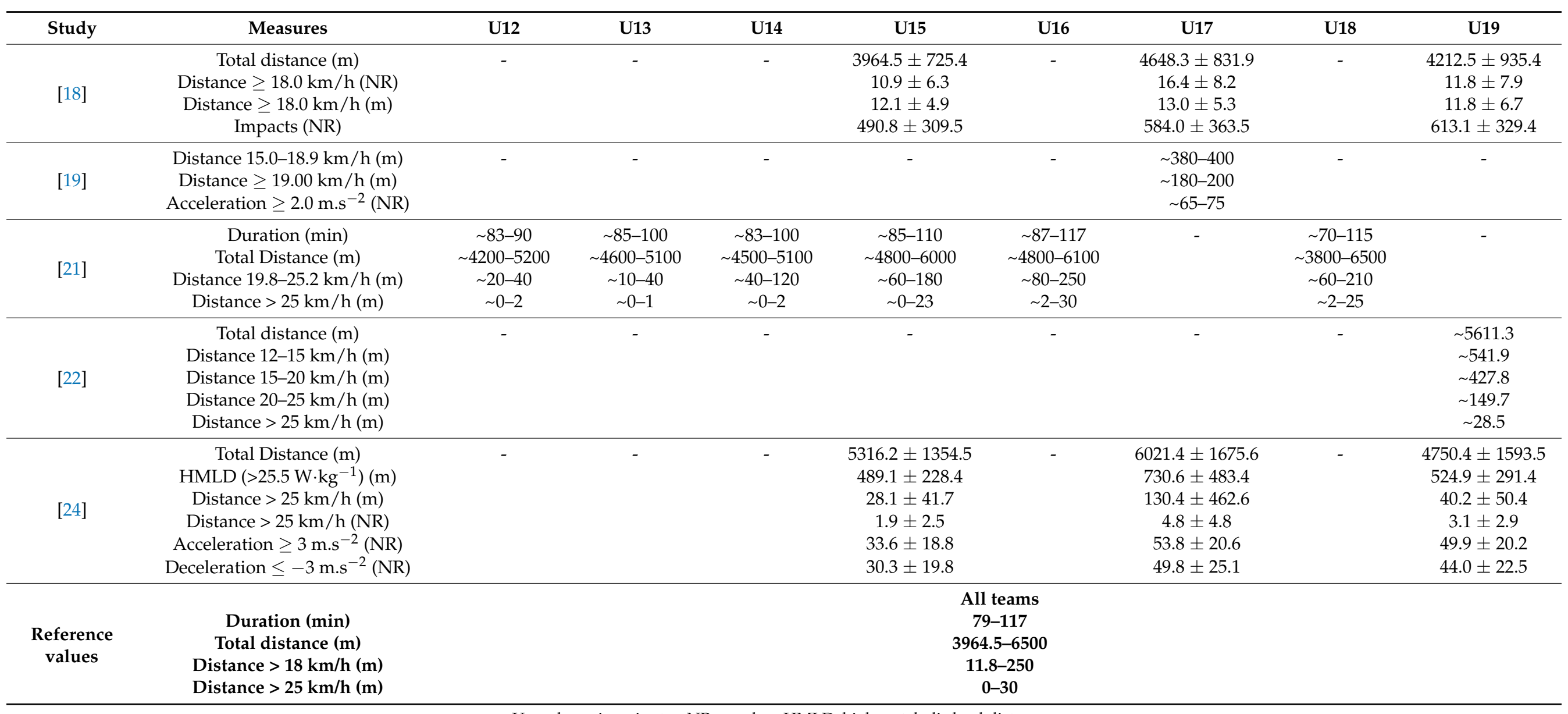

U: under; min: minutes; NR: number; HMLD: high metabolic load distance. 
Table 5. Results for external training intensity by match-day minus and by players positions.

\begin{tabular}{|c|c|c|c|c|c|c|}
\hline Study & Team and Measures & MD-5 & MD-4 & MD-3 & MD-2 & MD-1 \\
\hline$[21]$ & $\begin{array}{c}\text { U12 } \\
\text { Duration }(\mathrm{min}) \\
\text { Total Distance }(\mathrm{m}) \\
\text { Distance } 19.8-25.2 \mathrm{~km} / \mathrm{h}(\mathrm{m}) \\
\text { Distance }>25 \mathrm{~km} / \mathrm{h}(\mathrm{m})\end{array}$ & $\begin{array}{c}\sim 85 \\
\sim 4800 \\
\sim 30 \\
\sim 0\end{array}$ & $\begin{array}{c}\sim 83 \\
\sim 5200 \\
\sim 40 \\
\sim 0\end{array}$ & $\begin{array}{c}\sim 88 \\
\sim 4400 \\
\sim 30 \\
\sim 2\end{array}$ & $\begin{array}{c}\sim 90 \\
\sim 4200 \\
\sim 20 \\
\sim 1\end{array}$ & - \\
\hline$[21]$ & $\begin{array}{c}\text { U13 } \\
\text { Duration (min) } \\
\text { Total Distance }(\mathrm{m}) \\
\text { Distance } 19.8-25.2 \mathrm{~km} / \mathrm{h}(\mathrm{m}) \\
\text { Distance }>25 \mathrm{~km} / \mathrm{h}(\mathrm{m}) \\
\end{array}$ & $\begin{array}{c}\sim 100 \\
\sim 5100 \\
\sim 10 \\
\sim 0\end{array}$ & $\begin{array}{c}\sim 85 \\
\sim 4900 \\
\sim 40 \\
\sim 0\end{array}$ & $\begin{array}{c}\sim 85 \\
\sim 4700 \\
\sim 20 \\
\sim 0\end{array}$ & $\begin{array}{c}\sim 87 \\
\sim 4600 \\
\sim 30 \\
\sim 1\end{array}$ & - \\
\hline$[21]$ & $\begin{array}{c}\text { U14 } \\
\text { Duration (min) } \\
\text { Total Distance }(\mathrm{m}) \\
\text { Distance } 19.8-25.2 \mathrm{~km} / \mathrm{h}(\mathrm{m}) \\
\text { Distance }>25 \mathrm{~km} / \mathrm{h}(\mathrm{m})\end{array}$ & $\begin{array}{c}\sim 100 \\
\sim 5100 \\
\sim 60 \\
\sim 1\end{array}$ & $\begin{array}{c}\sim 85 \\
\sim 5100 \\
\sim 120 \\
\sim 2\end{array}$ & $\begin{array}{c}\sim 83 \\
\sim 4500 \\
\sim 40 \\
\sim 0\end{array}$ & $\begin{array}{c}\sim 90 \\
\sim 4800 \\
\sim 60 \\
\sim 0\end{array}$ & - \\
\hline$[21]$ & $\begin{array}{c}\text { U15 } \\
\text { Duration }(\mathrm{min}) \\
\text { Total Distance }(\mathrm{m}) \\
\text { Distance } 19.8-25.2 \mathrm{~km} / \mathrm{h}(\mathrm{m}) \\
\text { Distance }>25 \mathrm{~km} / \mathrm{h}(\mathrm{m})\end{array}$ & $\begin{array}{c}\sim 98 \\
\sim 5100 \\
\sim 60 \\
\sim 0\end{array}$ & $\begin{array}{c}\sim 110 \\
\sim 6000 \\
\sim 180 \\
\sim 23\end{array}$ & - & $\begin{array}{c}\sim 90 \\
\sim 5100 \\
\sim 110 \\
\sim 3\end{array}$ & $\begin{array}{c}\sim 85 \\
\sim 4800 \\
\sim 90 \\
\sim 1\end{array}$ \\
\hline$[21]$ & $\begin{array}{c}\text { U16 } \\
\text { Duration (min) } \\
\text { Total Distance }(\mathrm{m}) \\
\text { Distance } 19.8-25.2 \mathrm{~km} / \mathrm{h}(\mathrm{m}) \\
\text { Distance }>25 \mathrm{~km} / \mathrm{h}(\mathrm{m})\end{array}$ & $\begin{array}{c}\sim 93 \\
\sim 5000 \\
\sim 80 \\
\sim 2\end{array}$ & $\begin{array}{c}\sim 117 \\
\sim 6100 \\
\sim 250 \\
\sim 30\end{array}$ & - & $\begin{array}{c}\sim 90 \\
\sim 4900 \\
\sim 120 \\
\sim 10\end{array}$ & $\begin{array}{c}\sim 87 \\
\sim 4800 \\
\sim 80 \\
\sim 2\end{array}$ \\
\hline [21] & $\begin{array}{c}\text { U18 } \\
\text { Duration }(\mathrm{min}) \\
\text { Total Distance }(\mathrm{m}) \\
\text { Distance } 19.8-25.2 \mathrm{~km} / \mathrm{h}(\mathrm{m}) \\
\text { Distance }>25 \mathrm{~km} / \mathrm{h}(\mathrm{m})\end{array}$ & $\begin{array}{c}\sim 90 \\
\sim 4900 \\
\sim 105 \\
\sim 3\end{array}$ & $\begin{array}{c}\sim 115 \\
\sim 6500 \\
\sim 210 \\
\sim 25\end{array}$ & - & $\begin{array}{c}\sim 100 \\
\sim 6000 \\
\sim 200 \\
\sim 23\end{array}$ & $\begin{array}{c}\sim 70 \\
\sim 3800 \\
\sim 60 \\
\sim 2\end{array}$ \\
\hline
\end{tabular}


Table 5. Cont.

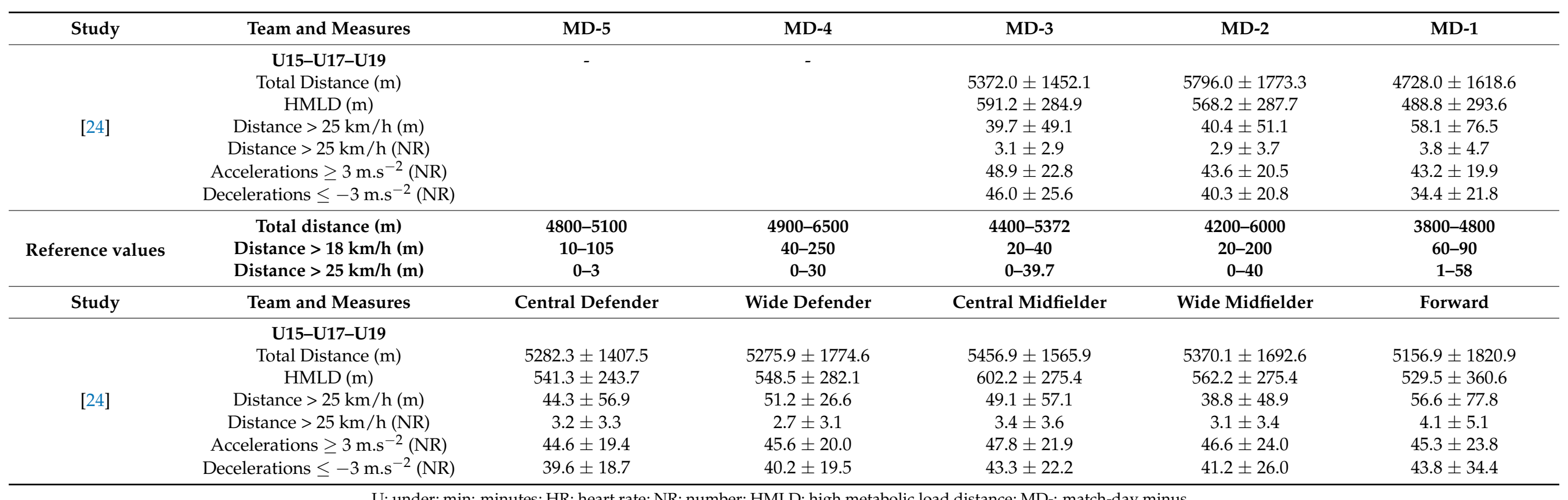

U: under; min: minutes; HR: heart rate; NR: number; HMLD: high metabolic load distance; MD-: match-day minus.

Table 6. Results for internal training intensity in one or two matches per week, by match-day minus and by player positions.

\begin{tabular}{|c|c|c|c|c|c|c|c|c|}
\hline \multirow{3}{*}{$\begin{array}{c}\text { Study } \\
{[20]}\end{array}$} & \multirow[t]{2}{*}{ Measures } & \multirow[t]{2}{*}{ Positions/Status/Team } & \multicolumn{3}{|c|}{ Training with 1-Match Week } & \multicolumn{3}{|c|}{ Training with 2 Matches-Week } \\
\hline & & & Session 1 & Session 2 & Session 3 & Session 1 & Session 2 & Session 3 \\
\hline & RPE (CR-10, au) & Overall U15 & $3.9 \pm 0.6$ & $3.5 \pm 0.9$ & $3.5 \pm 0.6$ & $3.4 \pm 0.6$ & $3.3 \pm 0.9$ & $2.3 \pm 0.5$ \\
\hline & s-RPE (CR-10, au) & Overall U15 & $157.1 \pm 42.2$ & $275.7 \pm 62.0$ & $283.0 \pm 47.8$ & $313.2 \pm 43.9$ & $293.9 \pm 71.1$ & $167.2 \pm 26.4$ \\
\hline
\end{tabular}


Table 6. Cont

\begin{tabular}{|c|c|c|c|c|c|c|c|c|}
\hline Study & Measures & Positions/Status/Team & \multicolumn{3}{|c|}{ Training with 1-Match Week } & \multicolumn{3}{|c|}{ Training with 2 Matches-Week } \\
\hline \multicolumn{9}{|c|}{ U17 } \\
\hline & & & MD-5 & MD-4 & MD-3 & MD-2 & MD-1 & - \\
\hline \multirow{5}{*}{ [23] } & \multirow{5}{*}{ s-RPE (CR-10, au) } & Wide defenders & $\sim 240$ & $\sim 360$ & $\sim 385$ & - & $\sim 150$ & - \\
\hline & & Central defenders & $\sim 245$ & $\sim 348$ & $\sim 390$ & - & $\sim 160$ & - \\
\hline & & Central midfielders & $\sim 243$ & $\sim 375$ & $\sim 400$ & - & $\sim 158$ & - \\
\hline & & Wide midfielders & $\sim 242$ & $\sim 380$ & $\sim 400$ & - & $\sim 158$ & - \\
\hline & & Forwards/strikers & $\sim 215$ & $\sim 355$ & $\sim 395$ & - & $\sim 152$ & - \\
\hline \multirow{2}{*}[24]{} & RPE (CR-20, au) & Overall U15-U17-U19 & - & - & $13.3 \pm 2.4$ & $12.5 \pm 1.7$ & $13.3 \pm 2.3$ & - \\
\hline & s-RPE (CR-20, au) & Overall U15-U17-U19 & - & - & $1196.1 \pm 211.2$ & $1158.1 \pm 221.2$ & $1194.4 \pm 205.2$ & - \\
\hline Study & & & Central Defender & Wide Defender & $\begin{array}{c}\text { Central } \\
\text { Midfielder }\end{array}$ & Wide Midfielder & Forward & - \\
\hline [23] & s-RPE (CR-10, au) & Overall U17 & $\sim 160-390$ & $\sim 150-385$ & $\sim 158-400$ & $\sim 158-400$ & $\sim 152-395$ & - \\
\hline \multirow{2}{*}{ [24] } & RPE (CR-20, au) & Overall U15-U17-U19 & 44.6 & 44.6 & 47.7 & 46.6 & 45.3 & - \\
\hline & s-RPE (CR-20, au) & Overall U15-U17-U19 & 261.2 & 230.5 & 265.3 & 238.1 & 255.9 & - \\
\hline \multirow{2}{*}{ [19] } & \multirow{2}{*}{ Banister TRIMP (au) } & U17 Starters & $\sim 105$ & - & - & - & - & - \\
\hline & & U17 Non-starters & $\sim 110$ & - & - & - & - & - \\
\hline \multicolumn{9}{|c|}{ Average training per overall team } \\
\hline \multirow{2}{*}{ [24] } & RPE (CR-20, au) & Overall U15 & $13.7 \pm 1.9$ & U17 & $15.5 \pm 1.8$ & U19 & $12.5 \pm 2.5$ & - \\
\hline & s-RPE (CR-20, au) & Overall U15 & $1235.3 \pm 171.9$ & U17 & $1215.5 \pm 158.7$ & U19 & $1120.2 \pm 224.7$ & - \\
\hline \multirow{3}{*}{ [25] } & RPE (CR-10, au) & U18 Gym training & $5.5 \pm 0.3$ & U18 Field Training & $6.6 \pm 0.6$ & - & - & - \\
\hline & RPE (CR-10, au) & U16 Gym training & $5.8 \pm 0.4$ & U16 Field Training & $6.3 \pm 0.4$ & - & - & - \\
\hline & RPE (CR-10, au) & U14 Gym training & $6.3 \pm 0.4$ & U14 Field Training & $6.2 \pm 0.2$ & - & - & - \\
\hline
\end{tabular}

U: under; min: minutes; MD-: match-day minus; au: arbitrary units; RPE: rated perceived exertion; s-RPE: session rated perceived exertion. 


\section{Discussion}

The aim of this systematic review was to identify and summarize studies that have examined external and internal training intensity monitoring in young male soccer players and to provide references values for the main training intensity measures. The main results showed the following range intervals by overall teams that include (U12 to U19):

training duration of 79-117 $\min [18,19,21,22,24]$;

total distance of 364.5-6500 m [18,19,21,22,24];

distance $>18 \mathrm{~km} / \mathrm{h}$ of $11.8-250 \mathrm{~m}[18,19,21,22]$;

distance $>25 \mathrm{~km} / \mathrm{h}$ of $0-30 \mathrm{~m}[18,19,21,22,24]$.

The importance of prescribing appropriate training intensities to improve player performance is well recognized in the current literature $[27,28]$. However, there remains a lack of clarity regarding the training intensity values most likely to promote improvements in young players performance.

\subsection{Reference Values Depending on Age Group}

When analyzing the mean of the total distance per training session for overall team of the selected studies, there was a pattern of increasing distance until older ages $[18,21,22,24]$ which was also corroborated by increase in the number of body impacts [18]. However, Dalen and Lorås reported a decrease in the number of accelerations from U15 to U17, respectively, [19], which was in line with Teixeira et al. that found the same pattern from U17 to U19 [24].

When considering, the accumulated weekly, total distance did not show that pattern from under U12 $(\sim 18.6 \mathrm{Km}), \mathrm{U} 13(\sim 19.3 \mathrm{~km}), \mathrm{U14}(\sim 19.5 \mathrm{~km}), \mathrm{U15}(\sim 21.0 \mathrm{~km}), \mathrm{U} 16$ $(\sim 20.8 \mathrm{~km}), \mathrm{U} 17(\sim 16.0 \mathrm{~km}), \mathrm{U} 18(\sim 21.2 \mathrm{~km})$ to U19 $(\sim 16.0 \mathrm{~km})$ age-groups $[18,19,21,22,24,25]$. Considering only the study of Hannon et al. that quantifying the training and match volume in male players from an English Premier League academy during two in-season microcycles, it was found an increase in the accumulative total distance from U12/13 $(38.3 \pm 5.1 \mathrm{~km})$, to U15 $(53.7 \pm 4.5 \mathrm{~km})$ and to U18 $(54.4 \pm 7.1 \mathrm{~km})$ [21]. Additionally, Abade et al. reported a higher total distance covered for U17 players than for U19 [18].

Perhaps technical-tactical methodologies, namely game model, can explain these data. There was an age-related increase in the training intensity and to a greater extent in the training volume [25]. Due to this fact, associated with a more conscious pacing strategy and better game interpretation with age, it was possible to also increase the exercise economics [24,29]. Even more interaction effects were found between inter-day and age [21,24], confirming an increase in the pacing strategy in the aging progression. Training periodization also seemed to influence the external intensity, concerning the training day and weekly microcycle [24].

The sprints number reported by the studies showed a high dispersion. In the study by Abade et al. [18] $11 \pm 6$ sprints (distance $=12 \pm 5 \mathrm{~m}$ ) were reported in U15 but in Teixeira et al. [24] only $2 \pm 3$ sprints (sprint distance $=28 \pm 42 \mathrm{~m}$ ), however the distance is twice that reported by Abade et al. [18]. In U17, the sprint number and sprint distance data were, respectively, between $16.4 \pm 8.2$ (sprint distance $=13.0 \pm 5.3 \mathrm{~m}$ ) [18] and $4.8 \pm 4.8$ (sprint distance $=130.4 \pm 462.6 \mathrm{~m}$ ) [24]. Teixeira et al. (2021) study evidenced the lowest intensity in U15 players' training sessions regarding high-speed run, average sprint distance, number of sprints [24]. The high standard deviation expresses the dispersion of results, which makes it difficult to standardize training intensity patterns related to the sprint number and distance at youth age.

Regarding internal training intensity, the main results showed a range interval range of 2.3 to 6.3 au for RPE between U14, U15, U16, and U18 [20,25] and 156 to 394 au for s-RPE between U15 and U17 [20,23]. Additionally, after conducting the research analysis of the present systematic review, a new study in U17 soccer players that analyze RPE and s-RPE measures was published [30]. That study [30] is in line with the interval range for RPE but higher values of 640 and 595 au were found for s-RPE during pre- and in-season with training durations around 96 and $95 \mathrm{~min}$, respectively, which may justify the higher values. 
Moreover, Wrigley et al. [25] noted a higher weekly RPE in the older age group (i.e., U18). However, the authors Teixeira et al. verified a higher training volume in younger players (i.e., U15 vs. U19) [24]. It is reasonable to argue that coaching team tends to code training programs with more volume and less intensity when it comes to younger players $[24,31,32]$. Furthermore, a focus on the basic tactical principles and technical skills using constrained training tasks was reported in younger age groups [25]. Nevertheless, the time spent at high-intensity zones and normalizing the session duration may affect the perceived exertion [33].

There were only one study analyzed Banister TRIMP and player status and found no differences between starters and non-starters [19] which was also corroborated by Martins et al. [30]. Nonetheless, it is important to reinforce that the period of the season and microcycle can influence result interpretations. For instance, the comparison of RPE between starters and non-starters showed significant differences in the following day after the match due to the recovery session for starters. Additionally, some differences were found during some mesocycles of the in-season [30].

\subsection{Training Intensity by Match Day Minus}

The main findings showed that young players usually training between 3 to 4 days per week. The higher intensity was found on MD-4, MD-3, and MD-2 while the lower intensity was found on MD-1, although only two studies used this approach for data analysis [21,24]. The previous findings were similar to adult players [34-39] which seems to be convergent in a tapering strategy based on a gradual reduction until the last day before the match [40].

The decrease in high-speed running distance and sprint distance in training sessions before match day was also evident [21] but it was not confirmed in sprint distance by Teixeira et al. [24]. Some studies reported that some coaches use sprinting and acceleration exercises for neuromuscular activation as a pre-match activation methodology $[24,40]$.

Regarding internal intensity analysis, two studies analyzed s-RPE with different scales, CR-10 [23] vs. CR-20 [24] and different training schedules, 4 [23] vs. 3 training sessions [24]. Even so, Nobari et al. found lower intensity in the following day of the match, higher intensity in mid-week, and the lowest intensities in the day before the match [23]. Although the study of Martins et al. [30] was in line with these findings, no differences between training days were found in Teixeira et al., study [24]. By contrast, previous studies reported an decreasing intensity phase in young players concerning RPE values [25,41] and Wrigley et al. (2012) evidenced a tapering in U18 players [25].

\subsection{Player Positions}

In youth soccer, the positional role has been analyzed in constrained training tasks [32,42,43], however, the present systematic review only found one study that analyzed intensity by position [24]. The study by Teixeira et al. analyzed three age-groups studied (U15, U17, U19) and reported that the greatest total distance was performed by the central midfielder $(5456.9 \pm 1565.9 \mathrm{~m})$, followed by the wide midfielder $(5370.1 \pm 1692.6 \mathrm{~m})$ [24]. The same authors only found significant differences between central defenders vs. forwards in highspeed running and sprint distance (minimum to moderate effect). Additionally, the internal training intensity presented significant differences between wide midfielder and forwards players (minimum effect). The same authors also reported an interaction effect between age, week, training day and playing position for deceleration [24].

The influence of playing position on physical and physiological performance during competition is well documented [44-46], while in training it seems to have a minimal effect for young players. In contrast, the influence of playing position in the adult training football has been well documented but it was non-conclusive [36,47-49]. On one hand, it was found greater training intensity for wide defenders and wide midfielders with respect to high-speed running and number of sprints when compared with the other positions [48]. In the same line, it was found in several mesocycles during an in-season, that central midfielders and wide midfielders displayed higher training intensity than 
central defenders with respect to total distance and high-speed running $\left(>19 \mathrm{~km} . \mathrm{h}^{-1}\right)$ [49]. Other study found that midfielders had the highest training weekly acute load of high metabolic load distance (6901 AU), while central defenders had the lowest (4986 AU) [47]. On the other hand, it was found no differences between players positions for total distance, high-speed running $\left(>19 \mathrm{~km} \cdot \mathrm{h}^{-1}\right)$ [36] in mesocycle and microcycle analysis during an in-season. The results of the present review seems unclear regarding the influence of player positions on training intensity. A possible explanation could be related to the training tasks, which may not be representative of the positional role specificity as referred by Ferraz et al. [29]. In future studies, the weekly training intensity quantification should consider the game model and representative game-based situations to promote playing position specificity. Furthermore, speed and acceleration thresholds in the studies of this review were based on elite gold-standard guidelines. Future research should focus on the adjustment thresholds for elite and sub-elite youth football.

In our literature review, only two studies reported values of the internal training intensity between playing positions [23,24]. Teixeira et al. Reported significant differences [24], however Nobari et al. found no differences in the comparisons within weekdays between the playing positions [23]. In addition, and Gjaka et al. also found no differences in accumulated weekly intensity between player positions [20]. If data from adult training were considered, the results seem to be in line because, it was found in several mesocycles during an in-season, that central defenders displayed higher training intensity than strikers or central midfielders with respect to s-RPE [49], while higher values of s-RPE were reported for midfielders than other positions [50]. On the other hand, no differences were between players positions for the same variable [36] in mesocycle and microcycle analysis during an in-season. This information revealed that a non-consensus still remain which suggest future studies to confirm the results.

\subsection{Study Limitations and Future Directions}

This study presents some limitations that influenced the results and the reference values provided. First, the small number of studies proves that much more research is needed in young players, even more considering the different age-group categories. Additionally, the studies included only analyze one soccer team which limit their results. Consequently, few studies analyzed contextual variables, such as player status, player positions, and MD- approach. In addition, few studies included full seasons. Moreover, the different speed thresholds and scales used makes difficult to generalize the results and compare them between studies. In addition, training intensity and reference values provided came from general training, but this information was not revealed by the studies. Only one study identify data from the field and from the gym training [25]. Finally, the present systematic review did not consider some possible inter-country variability or the context of each study which should be considered in future studies.

Despite the limitations, the present study constitutes a relevant tool in the field of training intensity quantification of young male soccer players that can be used for coaches, their staff and practitioners as a reference for future studies in order to replicate such values or even to increase the numbers presented.

In future studies, the comparison between elite and sub-elite soccer academies is an important research gap which should be considered. Additionally, information on what type of training and exercises could provide further knowledge on training intensity. At last, future studies can analyze values of training intensity for injury prevention, since the general aims of the studies in this field are to improve performance and avoid injuries.

\section{Conclusions}

The standard microcycle from U12 to U16 included three training sessions, while U17 and U19 included four training sessions per week. The duration of training sessions for all age-groups was between $\sim 70$ and $117 \mathrm{~min}$. 
Specifically, the following range intervals were found for training: RPE 2.3-6.3 au; s-RPE, 156-394 au; total distance, 3964.5-6500 $\mathrm{m}$ and; distance $>18 \mathrm{~km} / \mathrm{h}, 11.8-250 \mathrm{~m}$.

Even though the internal training intensity did not present significant differences for weekly inter-day analysis, there was an inverted U-shaped curve in the distribution of the weekly external training intensity. In the older age-group players, tapering is evidenced. On the other hand, the U14 to U16 players seems present relatively high training intensities across the weekly microcycle. Indeed, it could be suggested that coaches opt for different tapering strategies when the age and competition focus increases.

The playing position seems to have a minimal effect on the weekly training intensity of young players.

Author Contributions: Conceptualization, R.O. and F.M.C.; methodology, R.O., A.M.-V., M.R.-G. and F.M.C.; software, R.O., A.M.-V.; validation, R.O., J.P.B. and F.M.C.; formal analysis, R.O. and M.N.; investigation, R.O., J.P.B., A.M.-V., M.N. and F.M.C.; resources, R.O., J.P.B., A.M.-V., M.N. and F.M.C.; writing - original draft preparation, R.O., J.P.B., A.M.-V., M.N. and F.M.C.; writing-review and editing, R.O., J.P.B., A.M.-V., M.N. and F.M.C.; visualization, R.O., M.R.-G. and F.M.C.; supervision, R.O. and J.P.B.; project administration, R.O. All authors have read and agreed to the published version of the manuscript.

Funding: This research was funded by Portuguese Foundation for Science and Technology, I.P., grant number UIDP/04748/2020. The funders had no role in the design of the study, in the collection, analyses, or interpretation of data, in the writing of the manuscript, or in the decision to publish the results.

Institutional Review Board Statement: Not applicable.

Informed Consent Statement: Not applicable.

Data Availability Statement: Not applicable.

Conflicts of Interest: The authors declare no conflict of interest.

\section{References}

1. Weston, M. Training load monitoring in elite English soccer: A comparison of practices and perceptions between coaches and practitioners. Sci. Med. Footb. 2018, 2, 216-224. [CrossRef]

2. Staunton, C.A.; Abt, G.; Weaving, D.; Wundersitz, D.W.T. Misuse of the term 'load' in sport and exercise science. J. Sci. Med. Sport 2021. [CrossRef]

3. Rebelo, A.; Brito, J.; Seabra, A.; Oliveira, J.; Drust, B.; Krustrup, P. A New Tool to Measure Training Load in Soccer Training and Match Play. Int. J. Sports Med. 2012, 33, 297-304. [CrossRef] [PubMed]

4. Williams, A.M.; Reilly, T. Talent identification and development in soccer. J. Sports Sci. 2000, 18, 657-667. [CrossRef] [PubMed]

5. Impellizzeri, F.M.; Marcora, S.M.; Coutts, A.J. Internal and External Training Load: 15 Years On. Int. J. Sports Physiol. Perform. 2019, 14, 270-273. [CrossRef]

6. Halson, S.L. Monitoring Training Load to Understand Fatigue in Athletes. Sport. Med. 2014, 44, 139-147. [CrossRef] [PubMed]

7. Miguel, M.; Oliveira, R.; Loureiro, N.; García-Rubio, J.; Ibáñez, S.J. Load measures in training/match monitoring in soccer: A systematic review. Int. J. Environ. Res. Public Health 2021, 18, 2721. [CrossRef]

8. Scantlebury, S.; Till, K.; Sawczuk, T.; Weakley, J.; Jones, B. Understanding the Relationship Between Coach and Athlete Perceptions of Training Intensity in Youth Sport. J. Strength Cond. Res. 2018, 32, 3239-3245. [CrossRef]

9. Salter, J.; De Ste Croix, M.B.A.; Hughes, J.D.; Weston, M.; Towlson, C. Monitoring Practices of Training Load and Biological Maturity in UK Soccer Academies. Int. J. Sports Physiol. Perform. 2021, 16, 395-406. [CrossRef]

10. Becker, M.; Sperlich, B.; Zinner, C.; Achtzehn, S. Intra-Individual and Seasonal Variation of Selected Biomarkers for Internal Load Monitoring in U-19 Soccer Players. Front. Physiol. 2020, 11, 838. [CrossRef]

11. Maughan, P.C.; MacFarlane, N.G.; Swinton, P.A. Relationship Between Subjective and External Training Load Variables in Youth Soccer Players. Int. J. Sports Physiol. Perform. 2021, 16, 1127-1133. [CrossRef]

12. Akubat, I.; Patel, E.; Barrett, S.; Abt, G. Methods of Monitoring the Training and Match Load and Their Relationship to Changes in Fitness in Professional Youth Soccer Players. J. Sports Sci. 2012, 30, 1473-1480. [CrossRef] [PubMed]

13. Page, M.J.; McKenzie, J.E.; Bossuyt, P.M.; Boutron, I.; Hoffmann, T.C.; Mulrow, C.D.; Shamseer, L.; Tetzlaff, J.M.; Akl, E.A.; Brennan, S.E.; et al. The PRISMA 2020 statement: An updated guideline for reporting systematic reviews. BMJ $2021,372$. [CrossRef]

14. Rico-González, M.; Pino-Ortega, J.; Clemente, F.; Los Arcos, A. Guidelines for performing systematic reviews in sports science. Biol. Sport 2021, 39, 463-471. [CrossRef] 
15. Ryan, R.; Synnot, A.; Prictor, M.; Hill, S. Cochrane Consumers and Communication Group Data Extraction Template for Included Studies. Available online: http:/ / cccrg.cochrane.org/author-resources (accessed on 27 July 2021).

16. Slim, K.; Nini, E.; Forestier, D.; Kwiatkowski, F.; Panis, Y.; Chipponi, J. Methodological index for non-randomized studies (MINORS): Development and validation of a new instrument. ANZ J. Surg. 2003, 73, 712-716. [CrossRef] [PubMed]

17. Kim, S.Y.; Park, J.E.; Lee, Y.J.; Seo, H.-J.; Sheen, S.-S.; Hahn, S.; Jang, B.-H.; Son, H.-J. Testing a tool for assessing the risk of bias for nonrandomized studies showed moderate reliability and promising validity. J. Clin. Epidemiol. 2013, 66, 408-414. [CrossRef]

18. Abade, E.A.; Gonçalves, B.V.; Leite, N.M.; Sampaio, J.E. Time-motion and physiological profile of football training sessions performed by under-15, under-17, and under-19 elite portuguese players. Int. J. Sports Physiol. Perform. 2014, 9, 463-470. [CrossRef]

19. Dalen, T.; Lorås, H. Monitoring Training and Match Physical Load in Junior Soccer Players: Starters versus Substitutes. Sports 2019, 7, 70. [CrossRef]

20. Gjaka, M.; Tschan, H.; Francioni, F.M.; Tishkuaj, F.; Tessitore, A. Monitoring of Loads and Recovery Perceived During Weeks With Different Schedule in Young Soccer Players Spremljanje Obremenitev in Okrevanja Mladih Nogometašev V Več Tednih Z Različnimi Programi Treniranja. Orig. Artic. Kinesiol. Slov. 2016, 22, 16-26.

21. Hannon, M.P.; Coleman, N.M.; Parker, L.J.F.; McKeown, J.; Unnithan, V.B.; Close, G.L.; Drust, B.; Morton, J.P. Seasonal training and match load and micro-cycle periodization in male Premier League academy soccer players. J. Sports Sci. 2021, 39, 1838-1849. [CrossRef]

22. Houtmeyers, K.C.; Jaspers, A.; Brink, M.S.; Vanrenterghem, J.; Varley, M.C.; Helsen, W.F. External load differences between elite youth and professional football players: Ready for take-off? Sci. Med. Footb. 2020, 5, 1-5. [CrossRef]

23. Nobari, H.; Vahabidelshad, R.; Pérez-Gómez, J.; Ardigò, L.P. Variations of Training Workload in Micro- and Meso-Cycles Based on Position in Elite Young Soccer Players: A Competition Season Study. Front. Physiol. 2021, 12, 1-10. [CrossRef]

24. Teixeira, J.E.; Forte, P.; Ferraz, R.; Leal, M.; Ribeiro, J.; Silva, A.J.; Barbosa, T.M.; Monteiro, A.M. Quantifying sub-elite youth football weekly training load and recovery variation. Appl. Sci. 2021, 11, 4871. [CrossRef]

25. Wrigley, R.; Drust, B.; Stratton, G.; Scott, M.; Gregson, W. Quantification of the typical weekly in-season training load in elite junior soccer players. J. Sports Sci. 2012, 30, 1573-1580. [CrossRef] [PubMed]

26. Silva, P.; Dos Santos, E.; Grishin, M.; Rocha, J.M. Validity of heart rate-based indices to measure training load and intensity in elite football players. J. Strength Cond. Res. 2018, 32, 2340-2347. [CrossRef]

27. Bourdon, P.C.; Cardinale, M.; Murray, A.; Gastin, P.; Kellmann, M.; Varley, M.C.; Gabbett, T.J.; Coutts, A.J.; Burgess, D.J.; Gregson, W.; et al. Monitoring athlete training loads: Consensus statement. Int. J. Sports Physiol. Perform. 2017, 12, 161-170. [CrossRef]

28. McLaren, S.J.; Macpherson, T.W.; Coutts, A.J.; Hurst, C.; Spears, I.R.; Weston, M. The Relationships Between Internal and External Measures of Training Load and Intensity in Team Sports: A Meta-Analysis. Sport. Med. 2018, 48, 641-658. [CrossRef]

29. Ferraz, R.; Gonçalves, B.; Coutinho, D.; Oliveira, R.; Travassos, B.; Sampaio, J.; Marques, M.C. Effects of knowing the task's duration on soccer players' positioning and pacing behaviour during small-sided games. Int. J. Environ. Res. Public Health 2020, 17, 3843. [CrossRef]

30. Martins, A.D.; Oliveira, R.; Brito, J.P.; Loureiro, N.; Querido, S.M.; Nobari, H. Intra-season variations in workload parameters in europe's elite young soccer players: A comparative pilot study between starters and non-starters. Healthcare $2021,9,977$. [CrossRef]

31. Coutinho, D.; Gonçalves, B.; Figueira, B.; Abade, E.; Marcelino, R.; Sampaio, J. Typical weekly workload of under 15, under 17, and under 19 elite Portuguese football players. J. Sports Sci. 2015, 33, 1229-1237. [CrossRef]

32. Beenham, M.; Barron, D.J.; Fry, J.; Hurst, H.H.; Figueirdo, A.; Atkins, S. A Comparison of GPS Workload Demands in Match Play and Small-Sided Games by the Positional Role in Youth Soccer. J. Hum. Kinet. 2017, 57, 129-137. [CrossRef]

33. Haddad, M.; Stylianides, G.; Djaoui, L.; Dellal, A.; Chamari, K. Session-RPE method for training load monitoring: Validity, ecological usefulness, and influencing factors. Front. Neurosci. 2017, 11, 612. [CrossRef] [PubMed]

34. Clemente, F.M.; Owen, A.; Serra-Olivares, J.; Nikolaidis, P.T.; Van Der Linden, C.M.I.; Mendes, B. Characterization of the Weekly External Load Profile of Professional Soccer Teams from Portugal and the Netherlands. J. Hum. Kinet. 2019, 66, 155-164. [CrossRef]

35. Los Arcos, A.; Mendez-Villanueva, A.; Martínez-Santos, R. In-season training periodization of professional soccer players. Biol. Sport 2017, 34, 149-155. [CrossRef]

36. Oliveira, R.; Brito, J.P.; Martins, A.; Mendes, B.; Marinho, D.A.; Ferraz, R.; Marques, M.C. In-season internal and external training load quantification of an elite European soccer team. PLoS ONE 2019, 14, e0209393. [CrossRef] [PubMed]

37. Sanchez-Sanchez, J.; Hernández, D.; Martin, V.; Sanchez, M.; Casamichana, D.; Rodriguez-Fernandez, A.; Ramirez-Campillo, R.; Nakamura, F.Y. Assessment of the external load of amateur soccer players during four consecutive training microcycles in relation to the external load during the official match. Motriz. Rev. Educ. Fis. 2019, 25, e101938. [CrossRef]

38. Anderson, L.; Orme, P.; Di Michele, R.; Close, G.L.; Morgans, R.; Drust, B.; Morton, J.P. Quantification of training load during one-, two- and three-game week schedules in professional soccer players from the English Premier League: Implications for carbohydrate periodisation. J. Sport. Sci. 2016, 34, 1250-1259. [CrossRef] 
39. Oliveira, R.; Brito, J.; Martins, A.; Mendes, B.; Calvete, F.; Carriço, S.; Ferraz, R.; Marques, M.C. In-season training load quantification of one-, two- and three-game week schedules in a top European professional soccer team. Physiol. Behav. 2019, 201, 146-156. [CrossRef] [PubMed]

40. Brito, J.; Hertzog, M.; George, N. Do match-related contextual variables Influence training load in highly trained Soccer players? J. Strength Cond. Res. 2016, 30, 393-399. [CrossRef]

41. Impellizzeri, F.M.; Rampinini, E.; Coutts, A.J.; Sassi, A.; Marcora, S.M. Use of RPE-based training load in soccer. Med. Sci. Sports Exerc. 2004, 36, 1042-1047. [CrossRef]

42. Barnabé, L.; Volossovitch, A.; Duarte, R.; Ferreira, A.P.; Davids, K. Age-related effects of practice experience on collective behaviours of football players in small-sided games. Hum. Mov. Sci. 2016, 48, 74-81. [CrossRef]

43. Coutinho, D.; Gonçalves, B.; Santos, S.; Travassos, B.; Wong, D.P.; Sampaio, J. Effects of the pitch configuration design on players' physical performance and movement behaviour during soccer small-sided games. Res. Sport. Med. 2019, 27, 298-313. [CrossRef]

44. Pettersen, S.A.; Brenn, T. Activity Profiles by Position in Youth Elite Soccer Players in Official Matches. Sport. Med. Int. Open 2019, 3, E19-E24. [CrossRef]

45. Saward, C.; Morris, J.G.; Nevill, M.E.; Sunderland, C. The effect of playing status, maturity status, and playing position on the development of match skills in elite youth football players aged 11-18 years: A mixed-longitudinal study. Eur. J. Sport Sci. 2019, 19, 315-326. [CrossRef] [PubMed]

46. Aslan, A.; Açikada, C.; Güvenç, A.; Gören, H.; Hazir, T.; Özkara, A. Metabolic demands of match performance in young soccer players. J. Sport. Sci. Med. 2012, 11, 170-179.

47. Clemente, F.M.; Silva, R.; Ramirez-Campillo, R.; Afonso, J.; Mendes, B.; Chen, Y.S. Accelerometry-based variables in professional soccer players: Comparisons between periods of the season and playing positions. Biol. Sport 2020, 37, 389-403. [CrossRef] [PubMed]

48. Clemente, F.M.; Silva, R.; Castillo, D.; Arcos, A.L.; Mendes, B.; Afonso, J. Weekly load variations of distance-based variables in professional soccer players: A full-season study. Int. J. Environ. Res. Public Health 2020, 17, 3300. [CrossRef] [PubMed]

49. Oliveira, R.; Martins, A.; Nobari, H.; Nalha, M.; Mendes, B.; Clemente, F.M.; Brito, J.P. In-season monotony, strain and acute/chronic workload of perceived exertion, global positioning system running based variables between player positions of a top elite soccer team. BMC Sports Sci. Med. Rehabil. 2021, 13, 126. [CrossRef]

50. Clemente, F.M.; Mendes, B.; Nikolaidis, P.T.; Calvete, F.; Carriço, S.; Owen, A.L. Internal training load and its longitudinal relationship with seasonal player wellness in elite professional soccer. Physiol. Behav. 2017, 179, 262-267. [CrossRef] 